\title{
Opportunities for development of dynamic web interfaces in an information system for research projects
}

\author{
Plamen Milev ${ }^{*}$ \\ ${ }^{1}$ University of National and World Economy - Sofia, Bulgaria
}

\begin{abstract}
The article examines issues, related to the development of software solutions for project management. In this sense, the paper presents features of an information system for research projects. The realization of a dynamism of some of the user interfaces is a relevant issue for these software solutions. The main purpose of the article is to explore the opportunities for realization of such behavior and to present the relevant models for development of dynamic web interfaces in the information system through server-side implementation. To achieve its goal, the article uses various scientific methods, including study, analysis, research, modeling and experimentation. The results support the main hypothesis of the study, namely defining the benefits of development of dynamic web interfaces using server-side implementation. The conclusion highlights the opportunities for improvement of user experience in the information systems in the subject area through the application of the researched approach.
\end{abstract}

\section{Introduction}

Development of software solutions today involves the application of modern technological approaches. Most information systems are web based. Web-based information systems have several advantages over traditional software solutions. However, when it comes to controlling the user interface, traditional (desktop or native mobile) applications have an advantage. Their advantage is that at any point in the user's work with the application, the user interface can change depending on user behavior. This most often happens on the principle of timely processing of events. The reason is that the respective operating system intercepts the event and immediately forwards it to the application. In this way, the business logic of the application can change the user interface in cases where this is necessary. There are many examples of such a need. Very often such functionality is needed in the development of software solutions for project management. The reason usually lies in the fact that in these software solutions (as in many others) there are forms for filling in data for which it is not known in advance how much data will need to be entered. This is the case with data entry for projects by year. Different projects have different durations, which may not be known in advance. In relational databases, this issue is resolved with the use of primary and foreign keys. Another example is the entry of data for project participants. There are

*Corresponding author:pmilev@unwe.bg 
different numbers of participants in different projects. For this reason, the form for filling in the data must allow the fields for a new participant to be added dynamically. The other possibility is to enter data for a participant in an additional form. In traditional software solutions, both possibilities are relatively clear for implementation from a technological point of view. However, in web-based information systems, appropriate approaches need to be explored and applied. That is the purpose of the article.

\section{Technological approach}

Regarding the development of web-based information systems with variable (dynamic) user interfaces, attention should be paid to the various technological possibilities that can be applied. Unlike traditional software solutions, where this issue is not so relevant, web-based information systems have a client-side and server-side parts. In this sense, it is important to examine which part of the business logic for managing the user interface of the application is appropriate to implement client-side and which part is appropriate to implement serverside. There is a lot of research in this area that is not specifically related to the need for dynamic application behavior, but generally compares the client-side and server-side parts. Some authors give preference to server-side programming, but with the appropriate involvement of client-side in the need to process custom data requests [1]. Other authors emphasize the importance of the organization of business logic for user interface management in the overall digitalization process [2]. The issue has also been studied in the field of public services, where, in principle, there is a large set of forms in which the distribution of data is not known in advance [3]. Given the need for most modern software solutions to be web-based, the issues of development of dynamic web interfaces are extremely relevant. From the point of view of the possibilities for realization of such behavior in web-based information systems, different technological approaches can be applied. In the study we focus on the possibility of applying client-side together with server-side approach. As for the choice of client-side, JavaScript has almost no competition in the context of the issues of this study. JavaScript is an interpreted programming language, which is most wellknown as the scripting language for creating dynamic web pages [4]. There are a wide range of technological options for choosing a server-side. For the purposes of this study, the choice will be rather classic, namely ASP.NET. ASP.NET is a free web framework for building web applications [5]. ASP.NET offers different frameworks for creating web applications. For the purposes of the research, the concept of Web Forms will be applied. Web Forms are pages that users request using their browser [6]. Interactivity, which is inherent in traditional applications, requires a special approach in the development of web-based information systems. In the present study, ASP.NET Ajax is used to achieve interactivity [7]. After the selection of the appropriate technical means, a technological approach should be proposed, which will apply the selected technical means in a way that satisfies the purpose of the study. The proposal for an event processing process in the context of application of dynamic web interfaces is presented at Fig. 1 and includes the following elements:

- occurrence of an event at the client - usually this is a click of a button, after which the user expects additional dynamic fields to appear in the user interface to fill in data;

- preparation of a request - it is carried out with the use of JavaScript and Ajax, and for this purpose the current state of the dynamic elements in the user interface is established;

- making a request - asynchronous request from client-side to server-side part of the software solution;

- processing of the request from the server - after taking into account the current state of the web page, additional controls are prepared for the new dynamic fields for filling in data;

- return of a response - asynchronous response from server-side to the client-side part of the software solution; 
- updating the content at the client - the last step in the process in which the dynamic change in the web interface takes place.

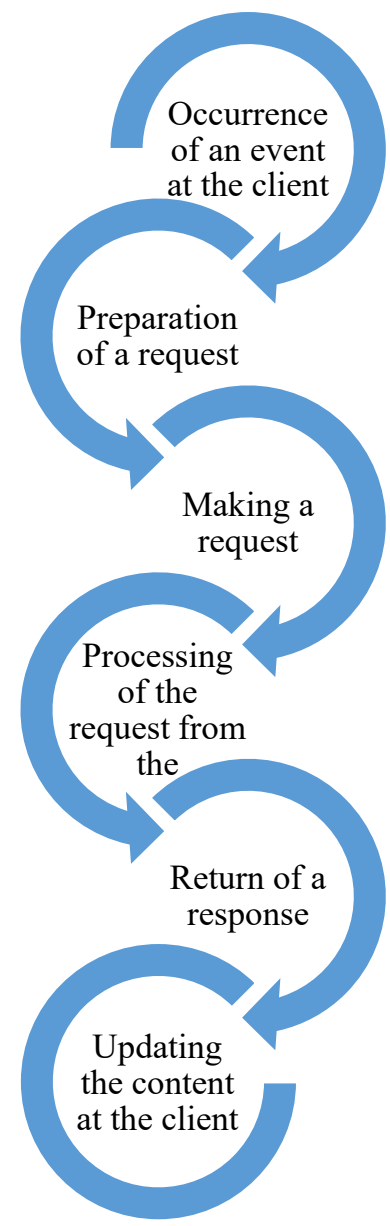

Fig. 1. Event processing process in the context of application of dynamic web interfaces

To analyze the applicability of the described technological approach, the results of its practical application for the development of dynamic web interfaces in an information system for research projects at UNWE should be examined [8].

\section{Results}

The information system for research projects is a web-based software solution for project management. As a result of the specifics of this type of software solutions, which were considered in the study, the information system has user interfaces, the content of which is not known in advance. The reason for this is that the specific content depends on the specifics of the project. That is why the technological approach proposed in the study has a very clear application in the context of developing an information system for research projects. This approach can be applied within several of the main groups of user interfaces of the system, including: 
- Web interfaces for project plan management, the years of which are not known in advance;

- Web interfaces for managing members of the project team, the number of which is not known in advance;

- Web interfaces for managing requests for machinery and equipment whose specification is not known in advance.

For the purposes of the present study, the results of the application of the proposed technological approach for the development of web interfaces for management of the project team members are presented. Given the uncertainty about the number of participants in the project, it is appropriate to apply the approach of dynamically adding controls to the information system. In this case, the occurrence of the event at the client is done by clicking the button, which is shown at Fig. 2.

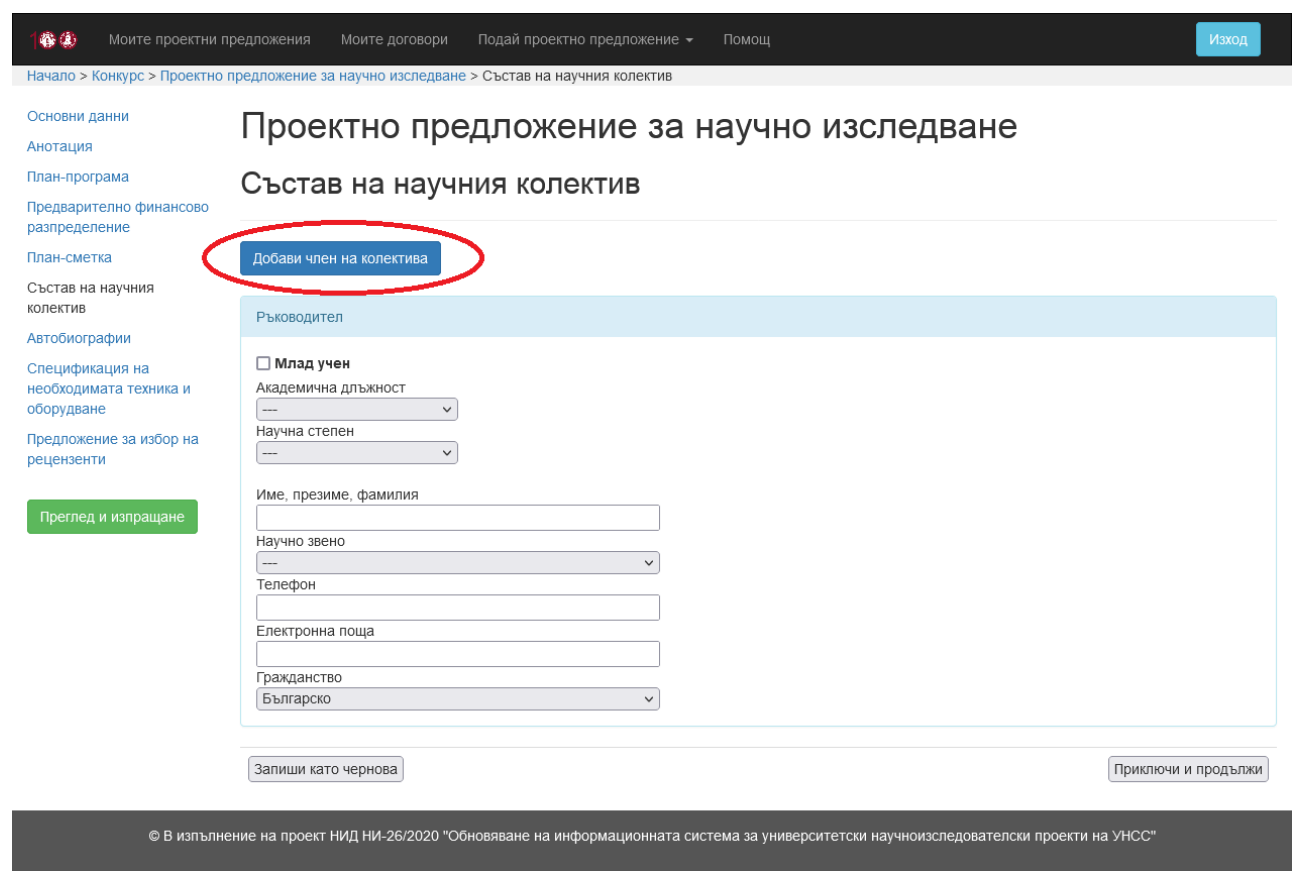

Fig. 2. Dynamic addition of content in an information system for research projects

After clicking the button "Add team member" the process goes through the steps described in the technological approach in the study. As a result, a group of fields for filling in data for an additional participant in the project is dynamically added to the web interface. The more times this button is clicked, the more groups of data fields will be dynamically added within the web interface in the information system for research projects. Usually, the need to dynamically add content is related to the opposite need - to dynamically remove content. If it is not possible to remove participants in the project, it will not be possible to claim that the developed web interfaces are dynamic. For this purpose, a customer event is provided, which is activated by clicking the button shown at Fig. 3. 
4) Моите проектни предложения Моите договори Подай проектно предложение - Помощ

Начало > Конкурс > Проектно предложение за научно изследване > Състав на научния колектив

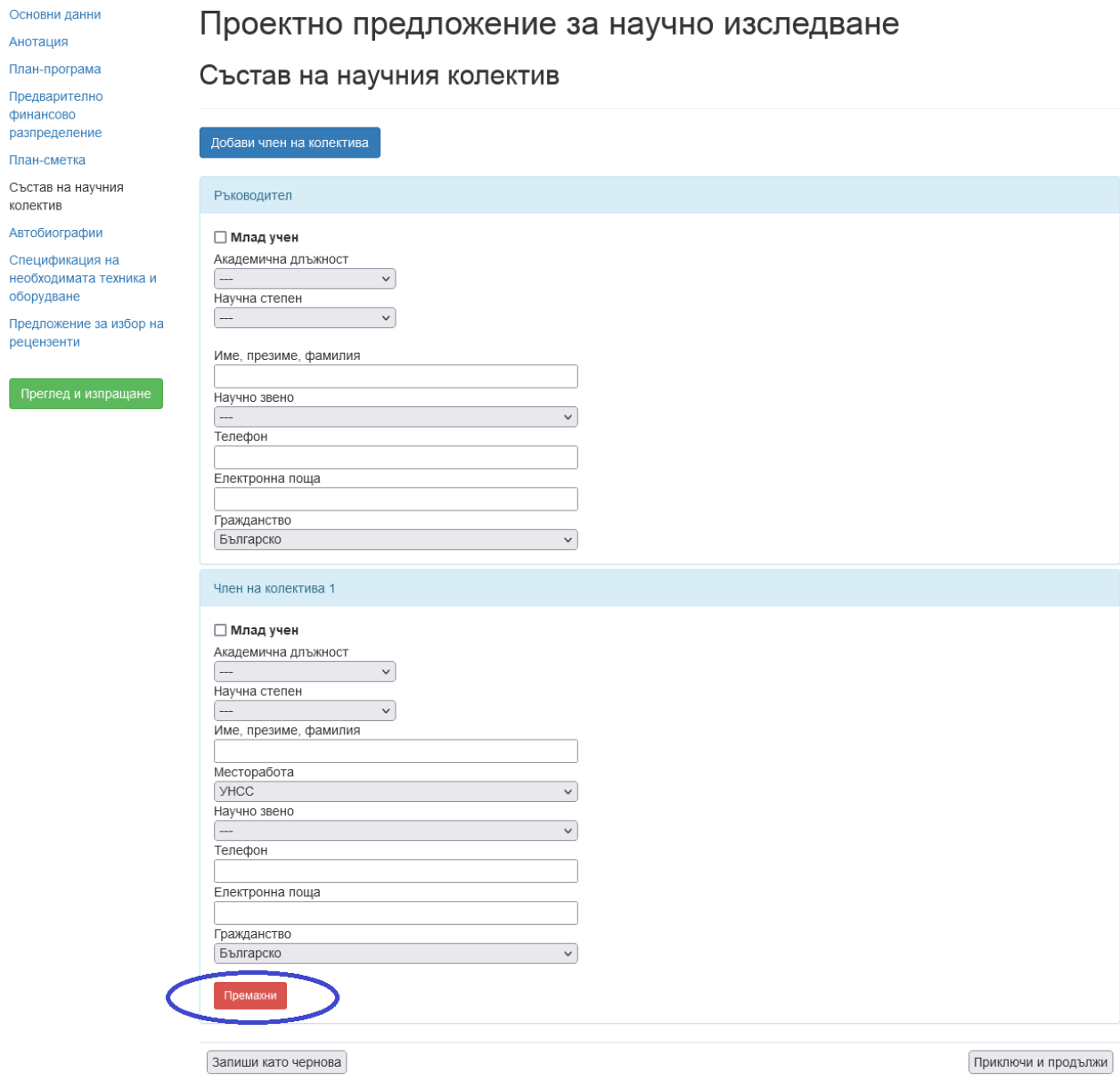

Fig. 3. Dynamic removal of content in an information system for research projects

The need for functionality to dynamically remove content from the user interface is as important as that to dynamically add content. In this sense, after clicking the "Remove" button for a specific project participant, its group of data fields will be dynamically removed from the user interface. Dynamic content removal goes through the same steps of a technological approach as dynamic content addition. From the point of view of communication between the client-side part and the server-side part of the software solution, it does not matter whether the operation selected by the system user is to add content or remove content. Both operations involve the application of the same approach from a conceptual and technological point of view.

\section{Conclusion}

As a result of the study, the following conclusions can be drawn: 
- there are a large number of concepts and technologies for the development of modern applications and in particular web-based information systems;

- when developing web applications, different approaches can be applied to create a user interface;

- very often, as in an information system for research projects, some elements of the user interface are not known in advance, which requires the development of dynamic web interfaces;

- development of dynamic web interfaces can be both client-side and server-side;

- The server-side approach has its advantages in cases where an asynchronous request model is used, which dynamically changes only part of the content of the web page.

The results of the research and the proposed technological approach find practical application in the development of an information system for research projects at UNWE.

This work was supported by the UNWE Research Programme (Research Grant No. NID NI-26/2020).

\section{References}

1. Client-side vs. Server-side vs. Pre-rendering for Web Apps (2020), https://www.toptal.com/front-end/client-side-vs-server-side-pre-rendering, [Accessed: 22 June 2021]

2. R. Kirilov, Econ. Altern. J., 1, 184-195 (2020)

3. K. Kirilova, Econ. and Soc. Alternatives, 2, 84-98 (2016)

4. JavaScript (2021), https://developer.mozilla.org/en-US/docs/Web/JavaScript, [Accessed: 22 June 2021]

5. ASP.NET overview (2019), https://docs.microsoft.com/en-us/aspnet/overview, [Accessed: 22 June 2021]

6. What is Web Forms (2014), https://docs.microsoft.com/en-us/aspnet/web-forms/whatis-web-forms, [Accessed: 22 June 2021]

7. ASP.NET Ajax: Enhanced Interactivity and Responsiveness (2009), https://docs.microsoft.com/en-us/aspnet/ajax/, [Accessed: 22 June 2021]

8. UNWE (2021), https://unwe.bg/, [Accessed: 22 June 2021] 